\title{
LOS SIGNIFICADOS DE LOS ALUMNOS HACIA LA ESCUELA SECUNDARIA EN MÉXICO
}

\author{
Inés Lozano Andrade \\ Escuela Normal Superior de México
}

\section{INTRODUCCIÓN}

La escuela es, a decir de Berger y Luckman (1986), una institución social que somete a los individuos a procesos diversos a fin de lograr la socialización y adaptación de los sujetos al sistema; aún más, para Foucault (1988), se asemeja a otras instituciones sociales como los reclusorios o sanatorios psiquiátricos, caracterizadas por el uso del panoptismo: todo se vigila, todo se castiga y además todo esto se hace "por el bien de los internos". Para Bourdieu (1995), es un espacio de reproducción de una cultura legítima a través de mecanismos de imposición violenta y arbitraria de formas de hacer y de ser. La escuela deviene así en una multitud de significados, pues por una parte, para los estudiosos críticos de esta, se asocia con violencia y represión, rodeada de panoptismo; pero para la sociedad en general hay una aceptación mayor hacia este último aspecto, aunque de manera acrítica, pues se concibe como una institución imprescindible para la formación en "bien del individuo y de la sociedad".

La escuela secundaria en particular se encuentra también en esta situación, pero más agudizada, en ella la disciplina se ha convertido en una obsesión a lograr, a decir de Giroux (1996), es un sitio donde el orden es la tarea y la contingencia el enemigo: donde un día bueno es un día de rutina, donde a la entrada y la salida hay personal vigilando y quizá esculcando los objetos de los estudiantes, donde hay personal especializado para vigilar, someter, canalizar a las instancias correspondientes a los infractores, donde los salones son fríos, los colores de las paredes deprimentes y las bardas, muy altas, donde el uniforme estricto es vital para poder acceder a la escuela, donde existen oficinas especiales para confrontar al estudiante que se ha salido de la norma y en la que se le asignan las sanciones: suspensiones temporales, tareas extras, trabajos físicos, llamadas de atención frente a los padres, todo tan revelador del carácter normativo de la escolarización.

Obvio es que en este contexto hay poder y dominio, pero no todos lo poseen legal $y / 0$ legítimamente, la sumisión es escalonada, el directivo tiene superiores, los superiores también; el maestro tiene al directivo y al programa, pero en esta escalera, el alumno es el que finalmente se encuentra en la parte más baja de los peldaños, es quien recibe las indicaciones del maestro, del plan de estudios, de las autoridades diversas y hasta de los padres.

A partir de esta realidad es como nos preguntamos sobre los significados de ser alumno, particularmente sobre cómo influyen en él las relaciones escolares que se construyen de manera simbólica cotidianamente y en particular, se abordan en esta investigación los aspectos de cómo se representan a la evaluación, por ser esta una parte medular del proceso educativo; en torno a la disciplina por ser esta una obsesión de la escuela secundaria; sobre los valores y su enseñanza por haberse convertido en un fin a conseguir por este nivel; $y$ finalmente, si todo esto tiene que ver con las asignaturas y profesores que les resultan más agradables. 
No es nuestra intención generar soluciones o propuestas, sino aportar elementos de análisis para formar un diagnóstico que considere a los actores educativos que están involucrados, actores que usualmente son usados para legitimar propuestas ya elaboradas o bien para simplemente sobrevivirlas, lo que afirma Contreras (1998) es fundamental en esto: en la educación, unos son los que deciden, otros son los que planifican y otros son los que ejecutan, nosotros agregamos lo siguiente: y otros son los que lo sobreviven.

También debemos aclarar que este no es un estudio con pretensiones de generalización o extensión del conocimiento, sabemos las limitaciones que una investigación cualitativa tiene; en efecto, el procedimiento metodológico que se siguió en este trabajo podemos caracterizarlo de la siguiente manera:

- Se parte de las intenciones de recuperar las representaciones sociales que se forman los alumnos en torno a la escuela secundaria en los aspectos antes mencionados, para lo cual empleamos la teoría de las representaciones sociales que originalmente Sergei Moscovici diera a conocer con su obra "El psicoanálisis, su imagen y su público", misma que otros autores van reconstruyendo paulatinamente. Con respecto a las representaciones sociales, Moscovici ha señalado que: "La representación social es una modalidad particular del conocimiento, cuya función es la elaboración de los comportamientos y la comunicación entre los individuos. La representación es un corpus organizado de conocimientos y una de las actividades psíquicas gracias a las cuales los hombres hacen inteligible la realidad física y social, se integran en un grupo o en una relación cotidiana de intercambios, liberan los poderes de su imaginación. (Moscovici, 1961. p. 17-18) A decir de Elejabarrieta (1991), las representaciones sociales están en todos lados y son necesarias para poder convivir con los demás miembros de un grupo, y puesto que el humano es un ser social que necesariamente requiere de los demás para poder subsistir, entonces siempre está en convivencia con grupos diversos con y para los cuales crea y recrea las representaciones sociales. Según Jodelet (2000. 10) "las representaciones sociales conciernen al conocimiento del sentido común, que se pone a disposición en la experiencia cotidiana; son programas de percepción, construcciones con estatus de teoría ingenua, que sirven de guía para la acción e instrumento de lectura de la realidad, sistemas de significaciones que permiten interpretar el curso de los acontecimientos y las relaciones sociales; que expresan la relación que los individuos y los grupos mantienen con el mundo y los otros; que son forjadas en la interacción y el contacto con los discursos que circulan en el espacio público; que están inscritas en el lenguaje y las prácticas, y que funcionan como un lenguaje en razón de su función simbólica y de los marcos que proporcionan para codificar y categorizar lo que compone el universo de vida". Así sabedores de que en la teoría mencionada existen modalidades diversas de análisis, propósitos distintos y metodologías diferentes, optamos por la tendencia interpretativa que, a nuestro parecer, da cuenta de la esencia de las representaciones sociales marcadas por las tres dimensiones que se mencionan como partes conformantes de las mismas: el campo de la representación, la actitud y la información, buscando en este estudio hacer un análisis dialéctico de estas tres dimensiones, sin separarlas artificialmente, sino presentándolas articuladas en la información recabada y analizada. 
- En efecto, hablar o estudiar este tema, desde estas intenciones, a la luz del positivismo resultaría intrascendente, pues implica analizar lo que no se ve, lo que no se mide, lo que no es comprobable y por tanto no es generalizable, sin embargo esto mismo es para otra vertiente de la ciencia, algo fundamental, pues se trata de rescatar los sentidos y significados del humano en su actuar social, es decir, conocer y rescatar la subjetividad inherente al ser humano; estamos entonces hablando de una línea de corte interpretativo que pretende comprender a los humanos y no explicarlos para después controlarlos. Se acepta en esta lógica que cada humano es diferente, porque ha vivido de manera diferente, en lugares diferentes, con personas diferentes, en tiempos diferentes, por lo tanto los sentidos de su vida y los significados que le otorga a lo que hace y cree, son diferentes y que si acaso hay similitudes entre aquellos que comparten espacios de tiempo, espacio y culturas similares: las personas en este mundo comparten una visión de su realidad, la construyen socialmente hablando porque esto les permite convivir con grupos, el humano es un ser gregario por necesidad y por naturaleza, pero también porque aislado enloquece. En los estudios cualitativo interpretativos, se trata de dar cuenta de esta subjetividad que permea en cada actor social y que comparten con los de su misma especie en contextos similares; para poder comprenderlos, hay entonces que, contextualizarlos, es decir dar cuenta de las condiciones en que se vive: los estudiantes de secundaria de ciertas zonas del Estado de México, conurbanas al Distrito Federal, no son los mismos, ni significan la escuela de la misma manera que otros, pues su contexto, su vida cotidiana, su historia personal no lo es. He ahí una limitante del estudio.

- Ahora bien, partiendo de estas premisas epistemológicas en las que la ciencia tradicional pierde su significado, se diseñaron las estrategias de investigación para acercarnos a las representaciones sociales de los alumnos de secundaria, para ello, se partió de la consignación de categorías teórico conceptuales emergidas de una diversidad de literatura revisada que daba cuenta de cada uno de los objetos en cuestión, de tal manera que estas categorías sirvieran como una primera guía para la aplicación de las técnicas de investigación de campo seleccionadas a fin de lograr el propósito de la investigación. Estas categorías conceptuales emergidas de un necesario marco teórico que siempre acompaña a todo investigador, una vez que se analizó la información de las entrevistas, fueron dando pautas a las categorías sociales que se encontraron en cada temática, las categorías sociales son constructos descriptivo interpretativos que dan cuenta de las coincidencias o incluso discrepancias de los informantes en torno al objeto que ocupa y siempre deben ser consideradas de manera contextual. Cada una de estas categorías sociales están desarrolladas en cada capítulo que conforma este trabajo.

- La entrevista semiestructurada fue la técnica seleccionada para recopilar la información durante esta investigación. La recolección de la información se realizó en el transcurso del ciclo escolar 2002-2003, para ello se delimitó el espacio geográfico en algunas secundarias de las dos principales modalidades que existen en la zona: secundarias generales y técnicas. Las escuelas seleccionadas se ubicaron en los municipios de Ecatepec, Tecamac y Zumpango, del Estado de México. En total se realizaron 57 entrevistas. 
- Una vez que se realizaron las entrevistas, se procedió a su análisis e interpretación, para lo cual se emplearon técnicas cualitativas de análisis de la información.

\section{ANÁLISIS DE RESULTADOS}

Los resultados de la investigación se presentan a continuación de manera resumida.

En torno a la evaluación, debemos decir que para cuando el adolescente se encuentra en la secundaria, ya ha vivido cuando menos seis años de experiencia escolar anterior, lo que genera en los estudiantes una serie de representaciones ya solidificadas, en las cuales el examen es visto como la forma predominante y mejor de ser evaluado, ante todo para satisfacer a los maestros y padres y al mismo tiempo como un indicador de la cantidad de conocimientos aprendidos. Emerge en ello una representación cuantitativa del saber, muy acorde al pensamiento hegemónico; sin embargo emergen también críticas a las formas inequitativas de evaluar: a algunos se les califica con mayor flexibilidad que a otros, esto se explica porque son los consentidos, los que siempre o mas bien casi siempre cumplen o quedan bien con el profesor, es decir, conocen el oficio del estudiante. No se percibe que los números sean la mejor forma de reflejar todo lo que se sabe, pero no se concibe otra forma de ser medidos sus conocimientos, así las propuestas de nuevas formas de evaluación se centran en: buscar que los conocimientos sean aplicados adecuadamente a situaciones prácticas, que el maestro considere todo el esfuerzo realizado a través de procesos de observación y que no se centre en un solo instrumento (el examen), además de que también se pueda evaluar al docente y sus prácticas de enseñanza. Las representaciones que se dan sobre la evaluación en la escuela secundaria en definitiva, están marcadas por la influencia decisiva de los padres en el proceso de escolarización, los alumnos prácticamente se representan la realidad escolar como sus padres les han pedido (exigido) que lo hagan: deben tener buenas calificaciones para poder demostrar lo que saben y ser alguien en la vida al continuar estudiando; deben ser evaluados con un instrumento neutral y objetivo: el examen, el cual certificará su saber; deben hacer lo que les diga el maestro o quedar bien con él; deben hacer lo posible por estudiar antes de los exámenes que se convierten en los momentos cumbres de la escolarización en la secundaria. Las representaciones de los alumnos responden a los sentidos y expectativas que los padres tienen acerca del futuro para ellos: que sean alguien en la vida y para ello, hay que estudiar más allá de la secundaria, para ello, hay que pasarla, sobrevivirla y de preferencia con buenas notas.

De igual manera que en lo anterior, ocurre con los valores y las representaciones que de ellos se forman los alumnos. Para los alumnos de secundaria indagados, tener valores es un sinónimo e comportarse bien en la escuela, portarse bien es a la vez tener respeto por los demás compañeros, incluso con los maestros y con ello, manifestar ser disciplinados, es decir estar apegados a la norma; finalmente hay aquí una percepción funcionalista y hegemónica de acomodación a la realidad normativa disciplinaria de la escuela que se refuerza con otras representaciones. Sin embargo se considera que los valores no pueden ser enseñados y por tanto aprendidos en la escuela, sino que estos vienen desde la familia en que se vive. Pareciera ser que la percepción que se tiene acerca de cómo se forman los valores desde el hogar implica una constante referencia al control de los impulsos y por tanto a la represión de los mismos por parte de los padres, que de esta manera demuestran su preocupación y atención a ellos o ellas, es decir, nuevamente emerge la idea de que la disciplina, como acatamiento de las normas, es señal inequívoca de poseer los valores adecuados al grupo o institución. También existe una representación crítica cuando se 
manifiesta que la escuela no siempre es un lugar en donde los alumnos pueden poner en práctica de manera libre esos valores aprendidos en el hogar. En la escuela, el "buen comportamiento", es decir el respeto de las normas escolares, puede darse por todo, por obligación, para no verse mal, etc., menos por convicción. Fuera de ella, al cesar la coerción, se actúa libremente, ya no se lleva la carga de la obligación, simplemente se actúa. A decir de los alumnos, la escuela no es quien debe inculcar valores, la escuela es apta únicamente para inculcar contenidos de las asignaturas. Además de esto, se percibe una incongruencia en las palabras y hechos de los maestros como una de las más importantes causas de lo que los propios docentes llaman falta de respeto. Los alumnos manifiestan que no se puede pretender enseñar valores cuando hay una sistemática falta de respeto o intolerancia entre otros aspectos entre los profesores hacia los alumnos. Los estudiantes por su parte no niegan la necesidad de los valores, pero no nuevamente impuestos como lo es casi todo en este nivel. La mayor parte de los esfuerzos porque los alumnos de escuela secundaria aprendan valores, se han limitado a la exposición oral y teórica.

Ahora bien, en continuidad con lo anterior, al averiguar acerca del significado que otorgan los estudiantes al concepto de disciplina; encontramos que es sinónimo de buen comportamiento y respeto, buen comportamiento en el sentido de respetar las normas establecidas en la institución como aceptables. En éste nivel de escolarización los alumnos tienen perfectamente claro qué es lo que se espera de ellos en cuanto a su comportamiento en la escuela, pues el modelo de alumno bien portado es el mismo en todos los ciclos educativos anteriores. El saber respetar es una característica del buen alumno, y un elemento de la disciplina dentro de la escuela. También se incluyen en la disciplina aspectos como la presentación, que consiste en portar adecuadamente el uniforme, el corte de pelo, el peinado, etc., y la puntualidad. El cumplimiento de esos aspectos viene a ser de suma importancia en la cotidianeidad de la escuela, hasta puede depender de ello el que se le permita o no la entrada a los alumnos. Los estudiantes manifiestan, sin embargo, una actitud de rechazo ante la exigencia excesiva de la disciplina, aunque piensan que es necesaria para mantener el orden, no están de acuerdo con la exageración en la presentación que se pide para permitir la entrada a la institución, es en las escuelas urbanas donde la revisión de éste aspecto es más rechazada por los adolescentes quienes incluso señalan que no hay relación entre su presentación y su aprendizaje. De todo esto, resulta ser una característica clara de la escuela secundaria, la obsesión por el control, el cual se logra únicamente con amenazas, reportes y regaños; las amenazas pueden ser, desde llevarlos a orientación hasta bajarles puntos o suspenderlos, pero éstas y los regaños no funcionan sino cuando provienen de los docentes que tienen la capacidad de ser estrictos para hacer respetar la disciplina, o que además de ser estrictos cuentan con la habilidad para dar bien su clase y despertar el interés del alumno. Este es un punto importante, alumnos indisciplinados afirmaron que cuando el tema es de su interés entonces están atentos, aunque también pueden simular que lo están cuando está de por medio su calificación. La simulación se utiliza, por alumnos conocidos como indisciplinados y de bajo aprovechamiento, como un medio para evitarse problemas con los maestros. En la escuela, los alumnos pueden ser identificados por sus compañeros y profesores, como inteligentes, flojos y/o desastrosos, según su comportamiento, lo cual le asigna a cada uno un papel determinado dentro de la clase, que es muy difícil que cambie, ya que la representación que el alumno tiene de sí mismo orientará su acción y entonces resultarán siempre los mismos los que hacen desorden, los que no cumplen con el trabajo escolar y los que participan y obtienen buenas calificaciones. Se construye en el proceso escolarizado un estigma que sella, que marca el futuro comportamiento del alumno, tanto de parte de los compañeros como de los profesores. Por otra parte, hay en las voces de los alumnos críticas a la manera en que se está diseñando y aplicando 
la disciplina en la secundaria, aunque estas se orientan más hacia el respeto por los aspectos propios de su apariencia que a las reglas de comportamiento internas para el aprendizaje. La disciplina en la escuela es vista por los alumnos como una forma de represión a sus inquietudes, por lo que se origina en ellos esa actitud de rechazo y de resistencia ante su imposición.

Respecto a las asignaturas que los alumnos de secundaria prefieren, son aquellas que prioritariamente implican desafíos intelectuales, a decir de ellos: Matemáticas, Física, Química y Biología. Con esto se comienza a resquebrajar la idea prejuiciada de que estas asignaturas y particularmente las Matemáticas, son consideradas como poco agradables, aburridas o muy difíciles. Un segundo factor que contribuye a formar una representación favorable hacia una asignatura, aparte de que promueva desafíos intelectuales, consiste en que debe haber una congruencia de los contenidos con la vida real, es decir, que debe haber una aplicación de los mismos a situaciones de la vida cotidiana, lo cual es difícil que el alumno lo pueda encontrar en asignaturas sociales o incluso las lenguas que se enseñan también en este nivel. Pero además de estos dos aspectos, parece que hay un tercer factor que interviene en el gusto por las mismas y se trata de la adecuada relación que el alumno establece con el profesor titular de la misma, la buena relación con el mismo es determinante para que su asignatura sea considerada de las preferidas.

Con relación al profesor destacado, los alumnos de secundaria coinciden con lo anterior, en una serie de atributos que puede poseer este tipo de profesor, que además a su favor esta el hecho de que debe trabajar en condiciones complejas; se presupone que estos rasgos fundamentales que son: el ser empático, el dominar los contenidos de la asignatura, el ser buen comunicador y el tener un adecuado manejo de la autoridad en el aula, se combinan si no todos si algunos de ellos en este profesor de tal manera que se considera que solo puede ser un buen comunicador si explica bien, si es sencillo, y ameno, y esto obviamente solo puede ocurrir si el profesor tiene rasgos de empatía, pues algo que se deplora en el ambiente estudiantil es la falta de tolerancia y respeto del profesor para con los alumnos; un docente que saluda a sus alumnos, los conoce por su nombre y les pregunta como están, es valorado, pero si además es respetuoso, y no abusa de su posición jerárquica, no pone apodos o se burla de ellos, es mucho más apreciado; pero si además de esto se interesa por sus problemas y les aconseja, es aún mejor valorado, lo consideran como un ser humano que se interesa por otros seres humanos en conflicto. Aunado a esto, este docente puede ser un buen comunicador y respetuoso, cuando se le plantean las dudas de la clase y él, $\sin$ desesperarse o molestarse, con paciencia y versatilidad en el discurso, puede explicar de formas distintas el tema del que se trate, lo cual va en conjunción con aquél aspecto de que plantea desafíos intelectuales para hacer atractiva la clase, porque solo alguien empático puede estar interesado en que el alumno aprenda con mecanismos diversos y creativos que impulsen y fomenten su pensamiento.

\section{CONCLUSIONES}

Podemos concluir que las representaciones sociales de los alumnos de secundaria, se forman en función de una serie de factores que se cruzan unos con otros y que complejizan las interpretaciones, pero no puede ser de otra manera si se trata de hacer un análisis más holístico de los fenómenos. Así podemos apreciar como una gran cantidad de dichas representaciones son prácticamente una herencia familiar y eventualmente de otros grupos, medios o instituciones. El hecho de que crean que no hay formas de evaluación diferentes, que consideren que el docente está plenamente autorizado y capacitado para ello, que ésta puede ser más bien justa y neutra a través de los exámenes sobre todo, que tiene valores quien se 
comporta bien y que quien lo hace así será recompensado tanto escolar como extraescolarmente, en una visión fatalista para aquellos "desadaptados" al sistema escolar que incluso llegan a justificar las sanciones que se toman contra ellos cuando no se "portan bien", pues portarse bien, en las voces más críticas, es hacer lo que te diga el maestro, en una actitud francamente conformista u oportunista e incluso hipócrita hacia los roles y normas; incluso cuando se cree que el mejor docente es aquél que mantiene el control del grupo, obsesión de la escuela secundaria. También se aprecia la influencia y aceptación de estas representaciones heredadas y aceptadas relativamente por los alumnos, cuando se habla de la necesidad de aceptar los valores y disciplina escolar para poder adaptarse en un futuro a la sociedad, pues quien no aprenda desde ese momento a seguir las normas, podrá fracasar en el futuro. Lo cual se ha convertido en un mito cada vez menos creíble, pues se habla de la crisis de la escuela como factor de movilidad social y esto los alumnos comienzan a percibirlo. De ahí que las representaciones mencionadas entre otras sean heredadas, pero no absolutamente compartidas, lo cual se evidencia en su comportamiento resistente en la escuela que aunque no llega a ser conciente y opositor, si está denotando inconformidad con lo que vive.

En efecto, la escuela, institución emergida en la modernidad, como aquella que dotaba de razón al individuo y que lo haría vivir mejor, haciendo a la vez que los demás vivieran mejor, pues con sus saberes podría controlar y transformar en su beneficio al mundo, hoy ha perdido en gran medida ese sentido original, pues las posturas posmodernas desacreditan o relativizan al saber científico y al poder de la escuela como mejoradora de la situación socioeconómica de los estudiantes. Aunado a esto, se genera con este devenir posmoderno una insatisfacción juvenil hacia la vida, una carencia de sentido hacia el futuro permea a las nuevas generaciones, no hay nada por que luchar o sentirse entusiasmado, más que el sentido de la riqueza, incluso a como de lugar; si un joven con un poco de talento puede triunfar y ganar mucho dinero (futbolista, artista, etc.), ¿qué importancia puede tener la escuela en este proceso?, ¿para qué estudiar tanto si la política o el narcotráfico en sus expresiones más extremas de movilidad social, pueden dar las satisfacciones que este capitalismo tardío promete? No hay ya una referencia de lo deseable y legítimo, los jóvenes bien pueden dedicarse a holgazanear, al fin y al cabo, nadie garantiza que si se esfuerzan en la escuela, habrá un futuro promisorio en lo económico siquiera; lo notan en los maestros que les rodean, quienes además, están sufriendo un malestar debido al deterioro de la imagen social del ser docente y a las condiciones laborales que viven cotidianamente, que son desalentadoras para esta profesión; el alumno no significa positivamente a la escuela de manera conciente, quizá solo lo hace como un reflejo de una representación colectiva y hegemónica que pretende vender la idea de que la escuela es aun un factor legítimo, de hecho sería uno de los pocos, para la mejora de su vida.

Si aunamos a este hecho que el alumno además cruza una etapa singular, socialmente construida como un momento de retraso a la madurez, a la incorporación a la independencia, donde se da una de rebeldía, una búsqueda constante de identidad, proceso en el cual, los mayores, pero mejor aun, la realidad virtual se convierte en fuente inacabable de modelos variados a seguir, al fin y al cabo, la posmodernidad se rige por el respeto a lo diverso, aunque no tenga ningún sentido humano y social; las utopías se han perdido para la juventud, solo queda el hedonismo y el relax del fin de semana, comenzando por el viernes y terminando el lunes. No es raro ya ver a algunos adolescentes de secundaria ebrios, drogados y organizando orgías en cualquier oportunidad, tampoco es difícil encontrar a algunos, los menos todavía, que por su situación particular en la que han vivido, se dedican a asaltar transeúntes, robar carros, vender droga o cuando menos fayuca. Finalmente ganan más dinero ahí que siendo profesionistas. La edad social 
del adolescente es ciertamente difícil, pero más difícil es porque la sociedad capitalista actual, no ofrece alternativas a estos millones de sujetos que no saben a que se van a dedicar o de que van a vivir más adelante.

Hace falta entonces, una reforma integral para la escuela secundaria, en donde se consideren estas voces, difícilmente consideradas y usualmente sometidas a los dictámenes de los especialistas y autoridades educativas, estas representaciones sociales que son denotativas de una situación crítica que es sentida, pero no reflexionada, donde lo que es evidente, es una carencia de significado a la escuela y sentido hacia la vida social desde lo que se haga en ella. En este sentido no es extraño que la secundaria en México tenga los peores resultados cuantitativamente hablando que cualquier otro nivel, los pocos estudios elaborados al respecto, así lo demuestran. Consideramos entonces que una reforma de este nivel, debe de tomar en cuenta el trabajar con las representaciones sociales de los diversos actores que en ella se encuentran, maestros, alumnos, directivos y padres de familia, ya que en todos ellos existen representaciones hegemónicas sobre este nivel que hay que concienciar y modificar para poder seguir adelante.

\section{BIBLIOGRAFÍA}

Berger, Peter y Thomás Luckman. La construcción social de la realidad. Amorrortu, Buenos Aires, Argentina, 1986

BouRdieu, Pierre. La reproducción. México, Paidós, 1990

Bourdieu, Pierre. Sociología y cultura. México, Grijalbo, 1995.

Casanova, Antonia. La evaluación educativa. México, SEP-Octaedro, 2000.

CONTRERAS Domingo, José. La autonomía del profesorado. Madrid, Morata, 1997.

ElejabarRieta, Francisco. "Las representaciones sociales". En: Echeverría, Agustín. Psicología social sociocognoscitiva. Desclee de Mowwer. Bilbao, España, 1991.

FOUCAULT, Michel. Vigilar y castigar. Nacimiento de la prisión. México, Siglo XXI. 1988.

GiRoux, Henry, “Educación posmoderna y generación juvenil”. Nueva Sociedad.. No. 146. Caracas, Edit. Texto. pp. 148-167, 1996

Giroux, Henry Y Mclaren, Peter. Sociedad, Cultura y Educación. Madrid, Instituto Paulo Freire, Miño Y Dávila Editores, 1996

GIROUX, Henry. Teoría y resistencia en educación. México, siglo XXI, 1992.

Habermas, Jurgen. Conocimiento e interés. Madrid, Taurus, 1992

HARGREAVES, Andy y otros. Una educación para el cambio. España. SEP-Octaedro, 2000

Hargreaves, Andy. Profesorado, cultura y posmodernidad. Morata, Madrid, 1996

IBÁÑEZ, Tomás. Ideologías de la vida cotidiana. Sendai, Barcelona, 1988.

JODELET, Denisse. "La representación social, fenómenos, concepto y teoría". En: Moscovici, Sergei. (1986). Psicología social. II. Pensamiento y vida social. México, Paidós, 1986. 
LozANo Andrade, J. I. Los saberes y cualidades pertinentes en la formación y práctica de los profesores de educación secundaria: un análisis desde sus representaciones sociales. Tesis de Doctorado, ENEP Aragón, UNAM, 2003.

Mclaren, Peter. Pedagogía crítica, resistencia cultural y la producción del deseo. Buenos Aires, Rei-Aique, 1993

MCLAREN, Peter. La escuela como un performance ritual. México, Paidós, 1994.

MCLAREN, Peter. La vida en las escuelas. México, siglo XXI, 1995

Moscovicl, Sergei. El psicoanálisis, su imagen y su público. Buenos Aires, Huemul, 1961

Moscovicı, Sergei. La psicología social. 2 tomos. Paidós, Barcelona,1982.

Quiroz, Rafael. "La educación secundaria en México al inicio del siglo XXI”. Educación 2001. (Marzo del 2001). Pp. 21-31

SACRISTÁN, Gimeno. El Currículum: Una reflexión sobre la práctica. Madrid, Morata, 1989.

SANDOVAL, Etelvina. Las tramas de la escuela secundaria. México. UPN-Plaza y Valdés, 2000

SANTOS Guerra. La evaluación: un proceso de comprensión, diálogo y mejora. Málaga, Aljibe, 2000.

Tedesco, Juan Carlos. "La educación y los nuevos desafíos de la formación del ciudadano". Nueva sociedad. No. 146. Caracas, Edit, Texto. pp. $74-89,1996$

TORRES Santomé, Jurjo. El Currículum Oculto. Madrid, Morata, 1991

YNCLÁN, Gabriela. (comp.). Todo por hacer: algunos problemas de la escuela secundaria. México, SNTE, 1999. 


\title{
Contactar
}

Revista lberoamericana de Educación

\author{
Principal OEI
}

